\title{
Análise do Conhecimento sobre a Assistência de Lesão por Pressão Entre um Grupo de Docentes do Curso de Enfermagem
}

\section{Knowledge Analysis about Pressure Injury Assistance among a Group of Nursing Course Professors}

\author{
Janaina Daniel Ouchi*a; Nádia Antônia Aparecida Polettib; Rodrigo Boscariola; Jeferson Agnellia \\ ${ }^{a}$ Faculdade Anhanguera. SP, Brasil. \\ ${ }^{b}$ Faculdade de Medicina de São José do Rio Preto. SP, Brasil. \\ *E-mail: janaina.ouchi@anhanguera.com
}

\begin{abstract}
Resumo
A profissão de enfermagem caminha rumo ao trabalho transdisciplinar, com formação humanizada e científica. A docência em enfermagem é uma prática desafiadora e tem tido destaque nas produções científicas no Brasil e no exterior, que ganhou destaque nas últimas duas décadas, em função da necessidade de docentes atualizados e que atendam as reformulações do currículo. Essa pesquisa teve como objetivo avaliar o conhecimento do docente enfermeiro a respeito de toda a Ciência acerca das diferentes lesões por pressão (LP). Foi realizado um estudo transversal, quantitativo, por meio da aplicação de um questionário investigativo, que analisou o conhecimento de docentes do Ensino Superior em uma Faculdade de Enfermagem da cidade de Sorocaba. Os docentes que participaram foram abordados pelo pesquisador, na instituição supracitada. As respostas dos enfermeiros participantes da pesquisa $(n=14)$ apontaram uma média de 43 acertos para as 46 questões aplicadas. Essas questões levaram em conta os critérios atualmente utilizados e que são considerados qualificadores da assistência de enfermagem. A enfermagem evoluiu consideravelmente, empoderando-se na tomada de decisões frente aos cuidados relacionados à lesão de pele, porém essa conduta ainda não faz parte da rotina da maior parte dos profissionais. Destaca-se a necessidade de atualização constante sobre o tema, pois o mercado oferece de forma contínua ferramentas que auxiliam na prevenção e tratamento das lesões de pele.
\end{abstract}

Palavras-chave: Lesão por Pressão. Educação em Saúde. Cuidados de Enfermagem. Medicina Preventiva.

\begin{abstract}
The nursing profession is moving towards transdisciplinary work, with humanized and scientific training. Nursing teaching is a challenging practice and has been highlighted in scientific productions in Brazil and abroad, which has gained prominence in the last two decades, due to the need for updated professors $s$ and who meet the curriculum reformulations. This research aimed to assess the nurse professor's knowledge regarding all the science concerning the different pressure injuries (LP). A cross-sectional, quantitative study was carried out by applying an investigative questionnaire, which analyzed the higher education professors' knowledge at a Nursing Faculty in the city of Sorocaba. The professors who participated were approached by the researcher at the aforementioned institution. The nurses'responses participating in the research $(n=14)$ indicated an average of 43 correct answers for the 46 applied questions. These questions took into account the criteria currently used and which are considered nursing care qualifiers. Nursing has evolved considerably, empowering itself in making decisions regarding care related to skin lesions, but this conduct is not part of the routine of most professionals yet. The need for constant updating on the topic is highlighted, as the market continuously offers tools that help in the prevention and treatment of skin lesions.
\end{abstract}

Keywords: Pressure Injury. Health Education. Nursing Care. Preventive Medicine.

\section{Introdução}

A enfermagem é uma profissão que está avançando ao trabalho multidisciplinar, com formação humanizada e científica, dando a possibilidade de sensibilizar os futuros profissionais a se tornarem enfermeiros com excelente desenvolvimento pessoal e profissional, além de preparar os discentes de enfermagem para os conhecimentos, habilidades, ética, moral e autoconhecimento (SANTAMARIA et al., 2019; TEO et al., 2019).

A docência em enfermagem é uma prática desafiadora e tem tido destaque nas produções científicas no Brasil e no exterior, que remete desde o início do século XX, porém ganhou destaque nas últimas duas décadas, em função da necessidade de docentes atualizados e que atendam as reformulações do currículo (MARTINS et al., 2019).

$\mathrm{O}$ ensino e aprendizagem na profissão do enfermeiro possui características únicas, por estar envolvido com a teoria e a prática, ensinar e aprender o cuidado coloca em pauta a eficácia do aprendizado, transforma os conhecimentos teóricos aprendidos em sala de aula em ações práticas de cuidado, sob a supervisão do docente (BETTANCOURT et al., 2011; MARTINS et al., 2020).

Os docentes não apenas precisam estar prontos para ensinar, mas necessitam atuar nas transformações dentro do processo de aprendizagem, em que os estudantes são instruídos e estimulados a tomada de decisões e aplicarem o que aprenderam em sala de aula na prática (CASTRO; PEREIRA, 2011).

A vida acadêmica exige vários cuidados durante toda aprendizagem, porém um desafio para enfermagem é o cuidado e a prevenção com as lesões por pressão (LP), recorrentes durante o período de hospitalização dos pacientes (DURRANT 


\section{et al., 2019; FEITOSA et al., 2020).}

Como educador, o enfermeiro possui a responsabilidade de capacitar seus alunos para orientar os pacientes e familiares nos cuidados. Cabe a esse profissional mostrar aos graduandos os desafios e as responsabilidades de como cuidar das lesões por pressão no período intra-hospitalar e após a alta e como lidar com todos os envolvidos (ALBUQUERQUE et al., 2019).

É imprescindível que os cuidados com as lesões de pele tenham continuidade e prolongamento, com tratamento embasado no conhecimento científico pelos profissionais de enfermagem, que assumem o papel de docente, pois a prática não é baseada apenas em evidências ou experiências vividas, mas aos conhecimentos e as características educacionais e individuais de cada ser humano (LOTFI et al., 2019; KIERSZENBAUM, 2012).

Vale ressaltar que a atuação da equipe de enfermagem deve estar voltada para a prevenção, sendo que na sua impossibilidade, deve auxiliar na restauração e contribuir para cura, assim como auxiliar o paciente no enfrentamento da moléstia, sempre atentando aos princípios científicos e tecnológicos (BLANES; FERREIRA, 2014; ZANEI; OLIVEIRA; WHITAKER, 2019).

As lesões por pressão (LP) são um grande problema de saúde no âmbito hospitalar, principalmente, nas Unidades de Terapia Intensiva (UTI) e nas instituições para idosos, em que o prolongado período que a pessoa permanece acamada se tornou uma das grandes razões que podem predispor os pacientes ao desenvolvimento de lesões por pressão (LOPES et al., 2019; MARTINS et al., 2020).

No Brasil, não existem dados consideráveis que indiquem a prevalência e a incidência das lesões por pressão, inúmeros são os cuidados de enfermagem voltados à prevenção da lesão por pressão ao paciente hospitalizado, pois os desenvolvimentos destas lesões durante o período de hospitalização elevam os custos e reduzem e muito a satisfação do paciente (GARRIGUES; CARTWRIGHT; BLISS, 2017).

Segundo a National Pressure Ulcer Advisory Panel (NPUAP), o European Pressure Ulcer Advisory Panel (EPUAP, 2010) junto com a Pan Pacific Pressure Injury Alliance (PPPIA), considera-se como Lesão por Pressão, qualquer dano localizado na pele ou em tecidos moles subjacente, em proeminência óssea ou causada por algum dispositivo médico, decorrente de atrito da pele em uma superfície na qual ocorre cisalhamento.

A tolerância dos tecidos, a isquemia depende de sua natureza e é influenciada pela estrutura da pele, a pressão é um dos principais fatores para o desenvolvimento das lesões por pressão, pois as fricções criadas pela força de duas superfícies provocam a remoção das células epiteliais (FEITOSA et al., 2020; ROSA et al., 2013).

Essas lesões ocasionam impactos para pacientes, famílias, sociedade e instituição de saúde. Além disso, são consideradas indicadores de qualidade na saúde, no que diz respeito às medidas implementadas durante o tratamento, mas principalmente, na prevenção (LOPES et al., 2019).

Considerando toda relevância do tema, esta pesquisa teve como objetivo avaliar o conhecimento do docente enfermeiro sobre toda a ciência acerca das diferentes lesões por pressão e, consequentemente, identificar as boas práticas assistenciais utilizadas pelos profissionais em campo de estágio.

\section{Material e Métodos}

Foi realizado um estudo transversal, quantitativo, por meio da aplicação de um instrumento de avaliação investigativo, que analisou o conhecimento de docentes do Ensino Superior em uma Faculdade de Enfermagem da cidade de Sorocaba.

\subsection{População e Amostra}

A amostra foi constituída por supervisores de estágio da faculdade de Enfermagem, que aceitaram participar da pesquisa, após assinatura do Termo de Consentimento Livre e Esclarecido (Apêndice I). A aplicação do instrumento de avaliação teve início após emissão do parecer consubstanciado do CEP pela Plataforma Brasil, com protocolo de número: 2.336.831.

A coleta das respostas foi realizada pelos pesquisadores, os supervisores participantes foram abordados no local de trabalho, com duração de aproximadamente 20 minutos. Foram excluídos os formulários, cujas respostas estiverem com duplicidade, que de alguma maneira comprometessem a compreensão da questão e dos supervisores que não aceitaram participar da pesquisa.

\subsection{Instrumento de pesquisa}

Utilizou-se um instrumento de avaliação elaborado por pesquisadores de uma Instituição de Ensino de Sorocaba em conjunto com pesquisadores da Faculdade de Medicina de São José do Rio Preto, baseado nos estudos de Ayello et al. (2017), Barakat-Johnson et al. (2018), França et al. (2019), contendo 46 questões fechadas, de respostas tipo verdadeiro (V) ou falso (F), que avaliaram conhecimentos de anatomia, fisiologia da pele, nomenclaturas, fatores de risco e intervenções de autonomia do enfermeiro.

Quadro 1 - Questões apresentadas aos participantes $(n=46)$.

\begin{tabular}{|c|l|c|c|}
\hline $\mathbf{N}^{\mathbf{0}}$ & \multicolumn{1}{|c|}{ Questões } & V & N/S \\
\hline 1 & O estágio I da lesão por pressão é definido como um eritema que não embranquece & & \\
\hline 2 & $\begin{array}{l}\text { Os fatores de risco para o desenvolvimento da lesão por pressão são: imobilidade, incontinência, nutrição } \\
\text { inadequada e alteração do nível de consciência }\end{array}$ & & \\
\hline 3 & $\begin{array}{l}\text { Todos os indivíduos em risco para lesão de pressão devem ter uma inspeção sistemática da pele pelo } \\
\text { menos uma vez por semana }\end{array}$ & \\
\hline
\end{tabular}




\begin{tabular}{|c|c|c|c|c|}
\hline $\mathbf{N}^{\mathbf{0}}$ & Questões & $\mathbf{V}$ & $\mathbf{F}$ & $\mathbf{N} / \mathbf{S}$ \\
\hline 4 & Água quente e sabonete podem ressecar a pele e aumentar o risco para lesão de pressão & & & \\
\hline 5 & É importante massagear as proeminências ósseas & & & \\
\hline 6 & Uma lesão de pressão de estágio II é uma perda parcial de pele envolvendo a epiderme e/ou a derme & & & \\
\hline 7 & $\begin{array}{l}\text { Todos os indivíduos devem ser avaliados na admissão no hospital quanto ao risco para desenvolver lesão por } \\
\text { pressão }\end{array}$ & & & \\
\hline 8 & $\begin{array}{l}\text { Amido de milho, cremes e curativos transparentes e curativos Hidrocolóides (tipo Duoderm ou Restore) não } \\
\text { protegem contra os efeitos da friçcão) }\end{array}$ & & & \\
\hline 9 & $\begin{array}{l}\text { Lesões por pressão no estágio IV apresentam uma perda de pele total com intensa destruição e necrose tissular } \\
\text { ou danos nos músculos, ossos ou estruturas de suporte }\end{array}$ & & & \\
\hline 10 & Uma ingestão dietética adequada de proteínas e calorias devem ser mantidas durante a doença & & & \\
\hline 11 & As pessoas que só ficam no leito devem ser reposicionadas a cada 3 horas & & & \\
\hline 12 & Uma escala com os horários de movimentação deveria ser escrita e colocada próxima do leito & & & \\
\hline 13 & Protetores de calcâneo como luva de água aliviam a pressão dos calcâneos & & & \\
\hline 14 & Rodas de água ou almofadas tipo argola auxiliam na prevenção da úlcera por pressão & & & \\
\hline 15 & Na posição lateral, a pessoa deve ficar em ângulo de $30^{\circ} \mathrm{com}$ a cama & & & \\
\hline 16 & $\begin{array}{l}\text { A cabeceira da cama deve ser mantida em baixo grau de elevação (de preferência não maior que um ângulo de } \\
30^{\circ} \text { ) consciente com as condições médicas }\end{array}$ & & & \\
\hline 17 & Uma pessoa que não pode se movimentar deve ser reposicionada enquanto sentada na cadeira a cada 2 horas & & & \\
\hline 18 & $\begin{array}{l}\text { As pessoas, que podem aprender, devem ser orientadas a mudar seu peso a cada } 30 \text { minutos enquanto sentadas } \\
\text { na cadeira }\end{array}$ & & & \\
\hline 19 & As pessoas, que permanecem na cadeira, devem ter uma almofada para proteção & & & \\
\hline 20 & Lesões de pressão no estágio II, apresentam uma perda de pele em sua espessura total & & & \\
\hline 21 & A epiderme deve permanecer limpa e seca & & & \\
\hline 22 & $\begin{array}{l}\text { A incidência de LP é tão elevada nos EUA que o governo americano criou uma comissão para estudar o risco, } \\
\text { a prevenção e o tratamento }\end{array}$ & & & \\
\hline 23 & Um ambiente de baixa umidade (seco) pode predispor a pessoa a LP & & & \\
\hline 24 & $\begin{array}{l}\text { Para minimizar a exposição da pele a umidade por incontinência, fraldas descartáveis e absorventes deveriam } \\
\text { ser usadas }\end{array}$ & & & \\
\hline 25 & A reabilitação deve ser instituída, se consistente com os planos gerais de terapia do paciente & & & \\
\hline 26 & Tecido desvitalizado é o tecido necrótico, amarelo ou creme no leito da ferida & & & \\
\hline 27 & A escara é boa para cicatrização da ferida & & & \\
\hline 28 & As proeminências ósseas não devem ficar em contato direto uma com a outra & & & \\
\hline 29 & $\begin{array}{l}\text { Toda pessoa avaliada como em risco para desenvolver LP deveria ser colocada em superfície (colchão) redutora } \\
\text { de pressão }\end{array}$ & & & \\
\hline 30 & Descolamento é a destruição que ocorre sob a pele & & & \\
\hline 31 & Escara é um tecido saudável & & & \\
\hline 32 & Embranquecimento refere-se à descoloração da pele quando a pressão é aplicada em uma área com hiperemia & & & \\
\hline 33 & Um colchão que alivia a pressão, reduz a pressão interface dos tecidos abaixo da pressão de fechamento capilar & & & \\
\hline 34 & A pele macerada pela umidade danifica-se mais facilmente & & & \\
\hline 35 & As LP são feridas estéreis & & & \\
\hline 36 & Uma cicatriz de LP poderá lesar mais rapidamente que a pele íntegra & & & \\
\hline 37 & Uma bolha no calcâneo não deve ser motivo de preocupação & & & \\
\hline 38 & Uma boa maneira de diminuir a pressão nos calcâneos é elevá-los do leito & & & \\
\hline 39 & Todo o cuidado administrado para prevenir ou tratar as LP precisam ser documentados & & & \\
\hline 40 & Cisalhamento é força que ocorre quando a pele adere a uma superfície e o corpo desliza & & & \\
\hline 41 & A fricção pode ocorrer ao movimentar a pessoa para cima na cama. & & & \\
\hline 42 & Baixo escore na escala de BRADEN está associado com um risco mais elevado para LP & & & \\
\hline 43 & A pele é o maior órgão do corpo & & & \\
\hline 44 & As LP de estágio II podem ser extremante doloridas pela exposição das terminações nervosas & & & \\
\hline 45 & $\begin{array}{l}\text { Para as pessoas que têm incontinência, a limpeza da pele deve ocorrer no momento que se sujar e nos intervalos } \\
\text { de rotina }\end{array}$ & & & \\
\hline 46 & Programas educacionais podem reduzir a incidência de LP & & & \\
\hline
\end{tabular}

V (verdadeiro), F (falso), N/S (não sabe)

Fonte: Dados da pesquisa.

\subsection{Análise dos dados}

Ao término da coleta dos dados, todos os questionários foram digitados e armazenados em uma planilha no Software
Excel 2016. Os dados foram submetidos a tratamento estatístico descritivo, através de frequências simples e porcentagens e analisados à luz dos achados da literatura científica específica do tema. 


\subsection{Aspectos éticos}

Todos os participantes, que concordaram em participar, assinaram o Termo de Consentimento Livre e Esclarecido de acordo com a Resolução 466/12 do Conselho Nacional de Saúde. Todos os gastos com esta pesquisa foram custeados pelos pesquisadores, não acarretando ônus de qualquer natureza para os participantes.

\section{Resultados e Discussão}

Após o período de aplicação do instrumento de pesquisa, quatorze supervisores de estágio concordaram em participar do estudo, o Quadro 2 descreve o número de acertos que cada questão do instrumento de avaliação obteve.

Quadro 2 - Número de acertos por questão obtidas pelos participantes da pesquisa $(n=46)$

\begin{tabular}{|c|c|c|c|c|c|}
\hline Questão & $\begin{array}{c}\text { Acertos } \\
\text { (n) }\end{array}$ & $\mathbf{\%}$ & Questão & $\begin{array}{c}\text { Acertos } \\
\text { (n) }\end{array}$ & $\mathbf{\%}$ \\
\hline 1 & 14 & 100 & 24 & 14 & 100 \\
\hline 2 & 10 & 71 & 25 & 13 & 93 \\
\hline 3 & 8 & 57 & 26 & 12 & 86 \\
\hline 4 & 9 & 64 & 27 & 14 & 100 \\
\hline 5 & 11 & 79 & 28 & 14 & 100 \\
\hline 6 & 11 & 79 & 29 & 10 & 71 \\
\hline 7 & 12 & 86 & 30 & 12 & 86 \\
\hline 8 & 14 & 100 & 31 & 8 & 57 \\
\hline 9 & 13 & 93 & 32 & 9 & 64 \\
\hline 10 & 9 & 64 & 33 & 11 & 79 \\
\hline 11 & 10 & 71 & 34 & 11 & 79 \\
\hline 12 & 14 & 100 & 35 & 12 & 86 \\
\hline 13 & 13 & 93 & 36 & 14 & 100 \\
\hline 14 & 12 & 86 & 37 & 13 & 93 \\
\hline 15 & 14 & 100 & 38 & 9 & 64 \\
\hline 16 & 11 & 79 & 39 & 14 & 100 \\
\hline 17 & 11 & 79 & 40 & 8 & 57 \\
\hline 18 & 12 & 86 & 41 & 10 & 71 \\
\hline 19 & 10 & 71 & 42 & 13 & 93 \\
\hline 20 & 14 & 100 & 43 & 14 & 100 \\
\hline 21 & 13 & 93 & 44 & 14 & 100 \\
\hline 22 & 9 & 64 & 45 & 14 & 100 \\
\hline 23 & 10 & 71 & 46 & 12 & 86 \\
\hline
\end{tabular}

Fonte: Dados da pesquisa.

Para a análise dos resultados foram estabelecidos quatro critérios: (a) definição dos indicadores, (b) alinhamento das medidas com as estratégias, (c) incorporação das medidas no trabalho e (d) aprendizagem através dos resultados. Observase, no Quadro 2, que das 46 questões aplicadas, 13 questões foram apontadas como V (verdadeira) pelos 14 docentes, sendo essas questões: 01, 09, 13, 16,20, 24, 27, 28, 36, 39, 44,45 , e 46.

Considerando a questão 1, em que se pergunta: o estágio I da lesão por pressão é definido como um eritema que não embranquece? Obteve-se $100 \%$ de acerto pelos participantes. Outra questão que obteve acerto dos 14 docentes se refere às Lesões por pressão no estágio IV se apresentam uma perda de pele total com intensa destruição e necrose tissular ou danos nos músculos, ossos ou estruturas de suporte. A resposta correta desta questão é V (verdadeira) e demonstra o conhecimento dos docentes quanto ao prejuízo tecidual que cada estágio da lesão acarreta ao paciente.

As intervenções de enfermagem devem ser reforçadas como a mobilização no leito, equipe multiprofissional interagindo no planejamento das atividades educativas, manejo da dor, elevação da cabeceira da cama até $30^{\circ}$, elaboração de programas de reabilitação de clientes com lesão medular, realização de pesquisas de enfermagem abordando fatores de risco, prevenção e novas terapias nas úlceras por pressão, conhecimento da prevalência das úlceras em hospitais gerais e uso de novas tecnologias na prevenção e tratamento (POLETTI, 2015; SAMPAIO, 2013).

Segundo Barakat-Johnson (2018), a qualidade da assistência de enfermagem é parte fundamental do processo de recuperação do paciente, sendo assim, a prescrição dos cuidados após a avaliação das necessidades do paciente faz toda diferença no processo de saúde e doença.

Consequentemente, a análise dos dados mostra que a questão 13 , que discute a utilização de protetores de calcâneo como luva de água alivia a pressão dos calcâneos, também obteve o acerto de todos os docentes que não utilizam deste método para proteção dos pacientes no leito.

Os conteúdos acerca da prevenção das úlceras por pressão enfocam questões relacionadas às medidas preventivas mais adotadas por enfermeiros, conhecimentos relacionados à utilização de programas de prevenção, com destaque das intervenções de enfermagem.

As demais questões que obtiveram $100 \%(16,20,24,27$, $28,36,39,44,45$, e 46) de acerto pelos docentes também estão ligadas diretamente com a assistência de enfermagem, considerando seu conhecimento sobre o tema imprescindível para o processo de cuidar.

Acredita-se que o enfermeiro deve estar em constante processo de atualização, para se apropriar de conhecimentos relacionados à assistência de enfermagem, adequar-se às suas finalidades essenciais e se motivar na busca da melhoria da qualidade (AYELLO, 2017; EDSBERG, 2016).

A prevenção necessita de uma estratégia individual precisa, o que exige muita dedicação e preparo do profissional, para uma intervenção de enfermagem ajustada e eficaz (FULBROOK et al., 2019; KREUTZ; SILVA, 2010).

Considerando todos participantes da pesquisa se obteve uma média de 43 acertos para as 46 questões aplicadas respectivamente, considerando todas como qualificadores da assistência de enfermagem. Durante a utilização do método de avaliação escolhido, estabeleceu-se a premissa de avaliação do conhecimento individual de cada docente, uma vez que o mesmo em campo de estágio deve se atentar para as condições gerais da pele: se está muito seca, desidratada, se há áreas de hiperemia, edema, se há áreas potenciais de ruptura, observar regiões como áreas sob aparelhos gessados, tração, talas, colares, entre outros (HERMIDA; ARAÚJO, 2016). 
Em virtude da complexidade que envolve a avaliação integral do paciente e a diversidade as competências exigidas pelos docentes, o Quadro 3 apresenta o número de acertos por supervisor que participou da pesquisa.

Quadro 3 - Número de acertos por questão obtida pelos supervisores de estágio $(n=14)$.

\begin{tabular}{|c|c|c|c|c|c|}
\hline Supervisor & $\begin{array}{c}\text { Acertos } \\
\text { (n) }\end{array}$ & $\mathbf{\%}$ & Supervisor & $\begin{array}{c}\text { Acertos } \\
\text { (n) }\end{array}$ & $\mathbf{\%}$ \\
\hline 1 & 43 & 93 & 8 & 45 & 98 \\
\hline 2 & 42 & 91 & 9 & 43 & 93 \\
\hline 3 & 40 & 87 & 10 & 42 & 91 \\
\hline 4 & 43 & 93 & 11 & 44 & 96 \\
\hline 5 & 45 & 98 & 12 & 45 & 98 \\
\hline 6 & 43 & 93 & 13 & 40 & 87 \\
\hline 7 & 44 & 96 & 14 & 41 & 89 \\
\hline & & & Média & $\mathbf{4 3}$ & $\mathbf{9 3}$ \\
\hline
\end{tabular}

Fonte: Dados da pesquisa.

Analisando o Quadro 3 se observa uma média de 43 acertos para as 46 questões aplicadas respectivamente, considerando todas como qualificadores da assistência de enfermagem. Observa-se ainda nos indicadores que nenhum dos quatorze supervisores participantes da pesquisa obtiveram $100 \%$ de acertos, sendo o menor número de acertos igual a 40 e o maior número de acertos igual a 45 .

Estudos demonstram que a prevenção de lesões por pressão não é uma prioridade para a enfermagem, porém como a afecção se apresenta como problema para o paciente, familiares e para a instituição de saúde, o enfermeiro deve conscientizar-se da importância de atuar em medidas profiláticas. Para que este cuidado seja eficaz, o profissional deve conhecer os mecanismos das lesões, bem como a realidade de instituição na qual trabalha (AYELLO et al., 2017; FEITOSA et al., 2020; FERNANDES, 2010).

A prevenção do desenvolvimento de lesões por pressão é uma atividade de responsabilidade do enfermeiro, pois a mudança de decúbito de $2 / 2$ horas faz parte da prescrição de enfermagem e deve ser seguida por toda a esquipe, sendo ele o profissional mais capacitado para lidar com esse problema (BLANES; FERREIRA, 2014; GEOVANINI, 2014).

As maiores dificuldades encontradas pela enfermagem envolvem a conscientização da equipe sobre a mudança de decúbito e o material adequado para a realização dos curativos específicos para cada estágio que a lesão se encontra.

Martins et al. (2020) e Pather (2016), em seus estudos, destacam que o conhecimento do enfermeiro é fundamental, pois o mesmo deve se atentar para o fato de que toda lesão que atinge determinado estágio se encontra em estágio de cicatrização, mesmo que apresente a formação de um novo tecido permanece no grau em que foi classificada, embora seja caracterizada como: em cicatrização.

Os resultados numéricos, descritos no Quadro 3, podem demonstrar a importância que a equipe de docentes tem a respeito da integralidade e o cuidado integral aos pacientes, ou seja, estão muito presentes nas práticas diárias dos profissionais em campo de estágio, além de serem considerados, por muitos autores, conceitos em constante construção em função dos vários sentidos que lhe são atribuídos no âmbito da saúde.

\section{Conclusão}

Os resultados evidenciam que grande parte dos fatores que propiciam as lesões, bem como os tipos de lesões de pele já fazem parte da rotina dos docentes participantes desta pesquisa. Considerando este resultado se pode afirmar que os principais pontos para investigação, diagnóstico e tratamento podem ser oferecidos pelo profissional em campo de estágio.

Destaca-se a necessidade de atualização constante sobre o tema, pois o mercado oferece, de forma contínua, ferramentas que auxiliam na prevenção e tratamento das lesões de pele, pois prestar um cuidado de qualidade a usuários com lesões de pele é um desafio a ser enfrentado por toda a equipe, em especial, pelo enfermeiro.

O potencial desse estudo é demonstrar que os educadores dos cursos de enfermagem devem preparar os discentes para o cuidado das lesões por pressão, com conhecimentos fisiopatológicos e demonstrando na prática os cuidados e a prevenção, visando o bem-estar e a vida do paciente.

\section{Referências}

ALBUQUERQUE, A. P. V. et al., O conhecimento dos enfermeiros assistenciais no tratamento de feridas. HU Rev. v.45, n. 3, p.295-298, 2019. doi: 10.34019/1982-8047. 2019. v45.28666.

AYELLO, A.P.A. et al. Educating Nurses in the United States about pressure injuries. Adv. Skin Wound Care, v.30, n.2, p.83-94. 2017. doi:10.1097/01.ASW.0000511507.43366.a.

BARAKAT-JOHNSON, M. et al. Knowledge and attitudes of nurses toward pressure injury prevention. J. Wound Ostomy Contin. Nur., v.45, n.3, p.233-237, 2018. doi: 10.1097/ WON.0000000000000430.

BETTANCOURT, L. et al. O docente de enfermagem nos campos de prática clínica: um enfoque fenomenológico. Rev. Latinoam. Enferm., v.19, n.5, p.1197-1204, 2011. doi: 10.1590/ S0104-11692011000500018.

BLANES, L; FERREIRA, L.M. Prevenção e tratamento de úlcera por pressão. São Paulo: Atheneu, 2014.

CASTRO, M.D.; PEREIRA, W.R. Cuidado integral: concepções e práticas de docentes de Enfermagem. Rev. Bras. Enferm., p.486-493, 2011. doi: 10.1590/S0034-71672011000300012.

DURRANT, L.A. et al. Health literacy in pressure injury: Findings from a mixed-methods study of community-based patients and carers. Nurs. Health Sci., v.21, n.1, p.37-43, 2019. doi: $10.1111 /$ nhs. 12429 .

EDSBERG, L.E. et al. Revised national pressure ulcer advisory panel pressure injury staging system: revised pressure injury staging system. J. Wound Ostomy Continence Nurs., v.43, n.6, p.585-597. 2016. doi: 10.1097/WON.0000000000000281.

EPUAP - European Pressure Ulcer Advisory Panel. National Pressure Injury Advisory Panel And Pan Pacific Pressure Injury Alliance. Prevention and Treatment of Pressure Ulcers/Injuries: quick reference guide. Europa: EPUAP/NPIAP/PPPIA, 2019

FEITOSA, D.V. S. et al. Atuação do enfermeiro na prevenção 
de lesão por pressão: uma revisão integrativa da literatura. Rev. Eletr. Acervo Saúde, v.43, n.43, p.2553, 2020. doi: 10.25248/reas. e2553.2020.

FERNANDES, L.M. Úlcera por pressão em clientes críticos hospitalizados: uma revisão integrativa da literatura. Ribeirão Preto: USP, 2000.

FULBROOK, P. et al. Australian Nurses' knowledge of pressure injury prevention and management: a cross-sectional survey. $J$. Wound Ostomy Contin. Nurs., v.46, n.2, p.106-112, 2019. doi: 10.1097/WON.0000000000000508.

FRANÇA, A.P.F.M. et al. Conhecimento de enfermeiros sobre o manejo de lesões por pressão em unidade de terapia intensiva. Rev. Eletr. Acervo Saúde, v.11, n.8, p.1-9, 2019. doi: 10.25248/reas. e576.2019.

GARRIGUES, L.J.; CARTWRIGHT, J.C.; BLISS, D.Z. Attitudes of nursing students about pressure injury prevention. $J$. Wound Ostomy Continence Nurs. v.44, n.2, p.123-128, 2017. doi:10.1097/WON.0000000000000302.

GEOVANINI, T. Tratado de Feridas Crônicas -Enfoque Multiprofissional. São Paulo: Rideel, v. 1, p 51-155, 2014.

HERMIDA, P.M.; ARAÚJO, I.E. Sistematização da assistência de enfermagem: subsídios para implantação. Rev. Bras. Enferm., v.59, n.5, p.675-679. 2016. doi: 10.1590/S003471672006000500015.

KIERSZENBAUM, A.L. Histologia e biologia celular: uma introdução à patologia. Rio de Janeiro: Elsevier; 2012.

KREUTZ, I.; SILVA, L. M. Pele: grande, importante e tão esquecida pelo enfermeiro. Rev. Pelle Sana. v.1, n.3, 2010.

LOPES, C. et al. Desenvolvimento de lesões por pressão em pacientes acamados: A percepção dos profissionais da área da saúde. Rev. Conhec. Online, v.3, p.143-157, 2019. doi: 10.25112/ rco.v3i0.1819.

LOTFI, M. et al. Iranian nurses' knowledge, attitude and behaviour on skin care, prevention and management of pressure injury: a descriptive cross-sectional study. Nurs. Open., v.6, n.4, p.1600-1605. 2019. doi:10.1002/nop2.365.

MARTINS, N. B. M. et al. Percepção de enfermeiros de terapia intensiva sobre prevenção de lesão por pressão. Rev. Atenção Saúde, v.18, n.63, p.43-45, 2020. doi:10.13037/ras. vol18n63.6270.

MARTINS, C. L. et al. O trabalho do enfermeiro enquanto docente do ensino superior: uma revisão narrativa. Rev. Eletr. Acervo Saúde, v.32, n.32, p.1159, 2019. doi:10.25248/reas. e1159.2019.

PATHER, A. P. et al. Best practice nursing care for ICU patients with incontinence-associated dermatitis and skin complications resulting from faecal incontinence and diarrhoea, International J. Evidence-Based Healthcare, v.14, n.1, p.15-23, 2016. doi:10.1097/XEB.0000000000000067.

POLETTI, N.A.A. O cuidado de enfermagem a pacientes com feridas crônicas: a busca de evidências para a prática. Ribeirão Preto: USP, 2015.

ROSA, T. J. S. et al. Úlceras por pressão: tratamento. Acta Fisiatr., v.20, n.2, p.106-111. 2013. doi:10.5935/0104-7795.20130017.

SAMPAIO, A. S.; RIVITTI, E. A. Dermatologia. São Paulo: Artes Médicas, 2012.

SANTAMARIA, N. et al. Preventing pressure injuries in the emergency department: Current evidence and practice considerations. Int Wound J. 2019, v.16, n.3, p.746-752, 2019. doi:10.1111/iwj.13092.

TEO, C.S.M. et al. Pressure injury prevention and management practices among nurses: A realist case study. Int. Wound J., v.16, n. 1, p.153-163, 2019. doi:10.1111/iwj.13006.

ZANEI, S.S.V.; OLIVEIRA, R.A.; WHITAKER, I.Y. Qualidade de vida dos profissionais de saúde dos programas de residência multidisciplinares. Rev. Enferm. UFSM. v.9, n.35, p.1-20, 2019. doi:10.5902/2179769230013. 